Supplementary Information for

\title{
Zinc Ions Modify Calcium Oxalate Growth by Distinct Transformation of Crystal Surface Termination
}

\author{
Bryan G. Alamani ${ }^{\ddagger \dagger}$, Julian D. Gale ${ }^{\S}$, and Jeffrey D. Rimer ${ }^{t, *}$ \\ $\$$ Department of Chemical and Biomolecular Engineering, University of Houston, 4726 \\ Calhoun Road, Houston, TX 77204, USA. \\ $\S$ Curtin Institute for Computation/School for Molecular and Life Sciences, Curtin University, \\ P.O. Box U1987, Perth, Western Australia 6845, Australia \\ $\dagger$ Department of Chemical Engineering, University of the Philippines Diliman, Quezon City \\ 1101, Philippines. \\ *Correspondence sent to: jrimer@central.uh.edu
}

\section{Table of Contents}

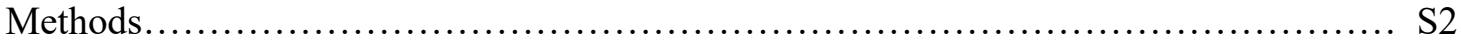

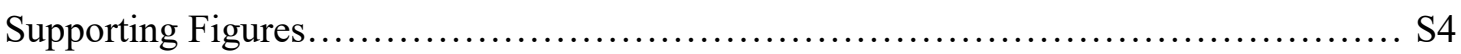

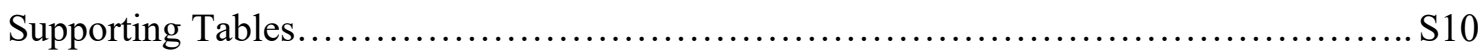

Movies.................................................................... S10

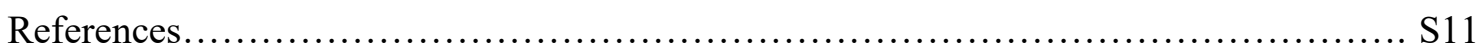

\section{List of Figures and Tables}

Figure S1. Invariant aspect ratio of COM crystals with increasing $\mathrm{Zn}^{2+}$ concentration

Figure S2. Average length of COM crystals at varying $\mathrm{Zn}^{2+}$ concentration

Figure S3. Optical micrographs of COM crystals extracted from bulk assays

Figure S4. Interstep distance on the COM (100) surface with increasing $\mathrm{Zn}^{2+}$ concentration

Figure S5. Bliznakov plot of step velocity data over a range of $\mathrm{Zn}^{2+}$ concentrations

Figure S6. Relative step velocity from AFM in growth solutions containing $10 \mu \mathrm{M} \mathrm{Zn}^{2+}$

Figure S7. AFM image of a COM (100) growth hillock in the presence of $20 \mu \mathrm{M} \mathrm{Zn}^{2+}$

Figure S8. Hypothetical COM terminations viewed along the (010) plane

Figure S9. Step heights on COM (100) surfaces in the presence of $\mathrm{Zn}^{2+}$ ions

Figure S10. Optimized structures for a zinc defect in different step sites on the COM (100)

Table S1. Defect energies at different sites on a COM (100) surface 


\section{METHODS}

Materials. The following reagents for COM crystallization were obtained from Sigma Aldrich (St. Louis, MO): calcium chloride dehydrate (ACS Reagent, 99+ \%), sodium oxalate (>99\%), sodium chloride $(>99.5 \%)$ and zinc chloride dihydrate $(>98 \%)$. All reagents were used as received without further purification. Deionized (DI) water, used in all experiments, was purified with an Aqua Solutions Type I RO DI filtration system (18.2 M $\Omega$ ).

COM Crystallization. Stock solutions of calcium chloride and sodium oxalate were prepared at $10 \mathrm{mM}$ concentration by dissolving corresponding quantities of each reagent in deionized water. Zinc chloride $(2 \mathrm{mM})$ and sodium chloride $(300 \mathrm{mM})$ stock solutions were prepared. A $10 \mathrm{~mL}$ solution of composition $0.7 \mathrm{mM} \mathrm{CaCl}_{2}: 0.7 \mathrm{Na}_{2} \mathrm{C}_{2} \mathrm{O}_{4}: 150 \mathrm{mM} \mathrm{NaCl}$ was prepared in a glass vial with varying amounts of $\mathrm{ZnCl}_{2}$. The quantity of $\mathrm{NaCl}$ was adjusted based on the concentration of $\mathrm{ZnCl}_{2}$ in order to maintain a constant overall ionic strength for the solution. The $\mathrm{Zn}^{2+}$ concentration ranged from $0 \mu \mathrm{M}$ (control) to $140 \mu \mathrm{M}$. All samples were incubated for $1 \mathrm{~h}$ at $60{ }^{\circ} \mathrm{C}$ prior to the dropwise addition of $10 \mathrm{mM} \mathrm{Na} 2 \mathrm{C}_{2} \mathrm{O}_{4}$ with constant stirring. All solutions were placed in an incubator at $60{ }^{\circ} \mathrm{C}$ for 3 days. A clean glass slide was placed at the bottom of the synthesis vial prior to incubation to collect crystals for analysis by microscopy.

Bulk Crystallization Assays. The dimensions of COM crystals along the [010] and [001] directions were measured by optical microscopy using a Leica DM2500-M microscope equipped with Olympus objectives. COM crystals collected on glass microscope slides were imaged in reflectance mode and recorded with a DM2500-M video camera. The [100] thickness of COM bulk crystals was assessed by scanning electron microscopy (SEM) using a FEI 235 Dual-Beam Focused Ion Beam instrument equipped with SEM sample extraction probe. COM crystals on glass slides were transferred to carbon tape by gently pressing the slide to the tape. Samples were coated with a ca. $15 \mathrm{~nm}$ layer of Au to reduce electron beam charging. In order to observe the time-resolved evolution of intergrowths in the presence of $\mathrm{Zn}^{2+}$, we removed samples at periodic times $(2,3$, and $5 \mathrm{~h})$ during COM bulk crystallization.

The kinetics of COM bulk crystallization was measured in situ using a calcium ionselective electrode (ISE) from Orion (model 9720BNWP). COM growth was analyzed at $23 \pm$ $2{ }^{\circ} \mathrm{C}$ with continuous stirring $(1200 \mathrm{rpm})$ using solutions of composition $0.5 \mathrm{mM} \mathrm{CaCl}: 0.5$ $\mathrm{mM} \mathrm{Na} 2 \mathrm{C}_{2} \mathrm{O}_{4}: 150 \mathrm{mM} \mathrm{NaCl}(\mathrm{S}=4.1)$ and varying concentrations of $\mathrm{ZnCl}_{2}$.

Atomic Force Microscopy (AFM). Samples were mounted on AFM specimen disks (Ted Pella, Inc.) covered with a thin layer of photocurable epoxy (Lens Bond Type SK-9). Crystals were transferred to the AFM sample by gently pressing the glass microscope slide on the partially cured epoxy (thermally cured in the incubator for $20 \mathrm{~min}$ ). The sample was placed back in the incubator for $1 \mathrm{~h}$ to fully cure the epoxy and anchor the COM crystals with either their (100) or (010) surface oriented in the plane of imaging. For in situ measurements of COM surface growth, we used either a Nanoscope IV (Digital Instruments, Santa Barbara, CA) or a Cypher ES (Asylum Research, Santa Barbara, CA) in contact mode at a scanning rate of 7 - 80 Hz. Growth solution with a supersaturation ratio $S=4$ was introduced into the AFM liquid cell at a rate of $0.2 \mathrm{~mL} \mathrm{~min}^{-1}$ using a dual syringe pump (CHEMYX Fusion 200) with an in-line flow mixing configuration that combines separate stock solutions of calcium and oxalate just prior to entering the liquid sample cell.

Density functional theory calculations. All first-principles calculations have been performed in the framework of Kohn-Sham density functional theory using the CP2K software ${ }^{1}$. Specifically, the Gaussian-augmented plane-wave approach was used ${ }^{2}$ in which triple-zeta double-polarized Gaussian basis sets are used in real space for the valence electrons for atoms, 
except for $\mathrm{Ca}$ and $\mathrm{Zn}$ where double-zeta polarized basis sets were employed due to their cationic nature. The atomic cores were represented by small core pseudopotentials of the Goedecker-Teter-Hutter ${ }^{3}$ form (i.e. $\mathrm{Ca}$ and $\mathrm{Zn}$ had 10 and 12 valence electrons, respectively). An auxiliary plane-wave basis set was used for the electron density with a cut-off of 600 Ry. The exchange-correlation functional used was $\mathrm{BLYP}^{4}$ with van der Waals corrections using the D3 method of Grimme and co-workers ${ }^{5}$. All calculations were performed at the gamma point, which is a good approximation for COM given that it is an insulator and a $2 \times 1 \times 1$ supercell was employed for the bulk such that all cell parameters exceed $10 \AA$.

The bulk monoclinic structure of COM was first relaxed at constant pressure, which resulted in unit cell parameters (corrected to the original cell in $a$ ) of $6.326 \AA(0.57 \%), 14.707 \AA$ $(0.85 \%), 10.241 \AA(1.23 \%), 90.00^{\circ}(0 \%), 109.92^{\circ}(0.4 \%), 89.53^{\circ}(-0.5 \%)$, where percentage changes relative to the experimental cell ${ }^{6}$ are given in parenthesis. As expected for a GGA functional, there is a systematic overestimation of the cell volume by a small amount. Following bulk relaxation, the (100) surface was created using the GDis code ${ }^{7}$, initially as a 2D slab with a thickness of 4 layers $(\sim 23.7 \AA)$ containing 320 atoms within the cell. This was then converted to 3-D periodic boundary conditions with a vacuum gap between images of 20 $\AA$. For all subsequent surface calculations the unit cell was held fixed while all internal coordinates were optimized. As reported in the main text, the computed surface energy for this configuration with steps in the [001] direction is $0.564 \mathrm{~J} / \mathrm{m}^{2}$. This was chosen in preference to the alternative configuration with steps in [010] which has a slightly higher surface energy of $0.614 \mathrm{~J} / \mathrm{m}^{2}$.

During the calculation of the bulk defect energies for zinc and magnesium substitution, a single $2 \times 1 \times 1$ supercell was used with full relaxation of the unit cell to minimize any strain effects. To compute the energy of $\mathrm{Zn}^{2+}$ and $\mathrm{Mg}^{2+}$ relative to $\mathrm{Ca}^{2+}$ a single point calculation was used in a fixed unit cell such that the charge-neutralizing background energy cancels out. Hydration free energies to correct the thermodynamics to the aqueous state were taken from the work of Marcus ${ }^{8}$.

The set of defect energies for $\mathrm{Zn}^{2+}$ substitution of $\mathrm{Ca}^{2+}$ for the possible outer layer sites near the step edges are given in Table S1 with the corresponding geometries shown in Figure S10. All of the sites of the COM crystal surface, with the exception of one (site 3), are more stable than the endothermic bulk replacement of calcium by zinc. In the case of the site labelled number 3, the $\mathrm{Zn}$ atom is situated almost outside of the step, and so while the species to which it is coordinated are similar to other sites, they are arranged largely on one side of $\mathrm{Zn}$, which accounts for the lower stability. Inclusion of waters of hydration may lower the energy of this site more than others due to the greater exposure of $\mathrm{Zn}$ to the external surface. The other point that is an outlier is site number 1 , which is more stable than the others; however, in this site $\mathrm{Zn}$ has reacted with water to form a $\mathrm{Zn}(\mathrm{OH})^{+}$complex, leading to protonation of a nearby oxalate anion. Again, inclusion of hydration of the surface is likely to suppress this reaction, which would likely reduce the exothermicity of this site. Full atomic positions for the defects are provided as PDB files. 


\section{SUPPORTING FIGURES}

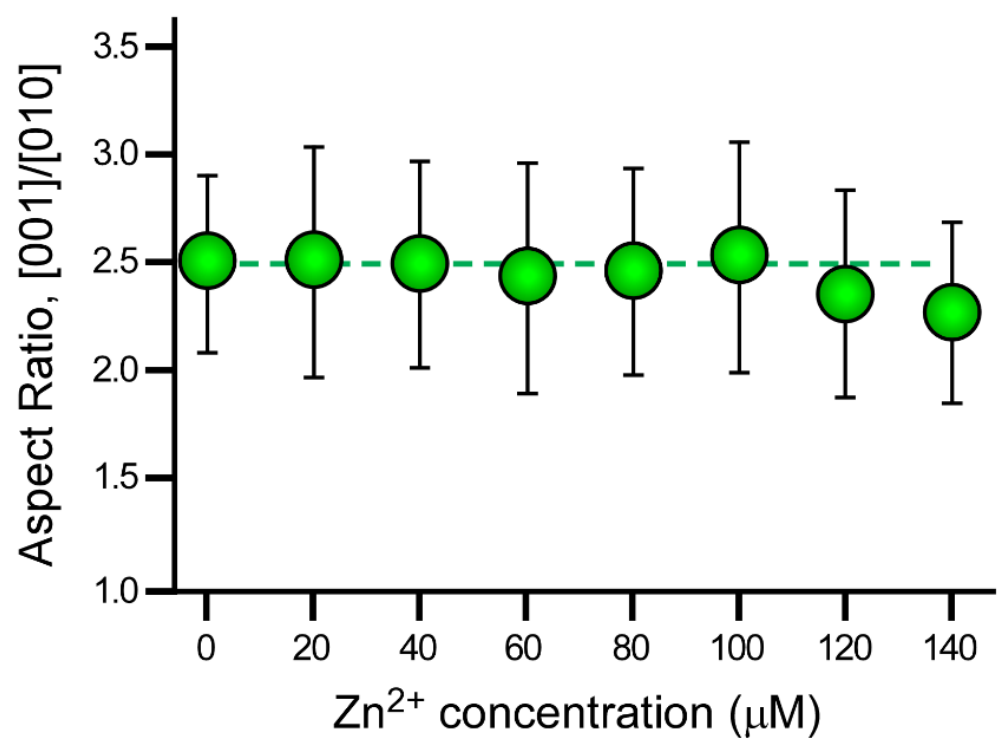

Figure S1. Constant aspect ratio of COM crystals in the presence of $\mathrm{Zn}^{2+}$ ions. Symbols are the average of more than 500 measurements from a single batch. Error bars span two standard deviations. The dashed line is interpolated to guide the eye.

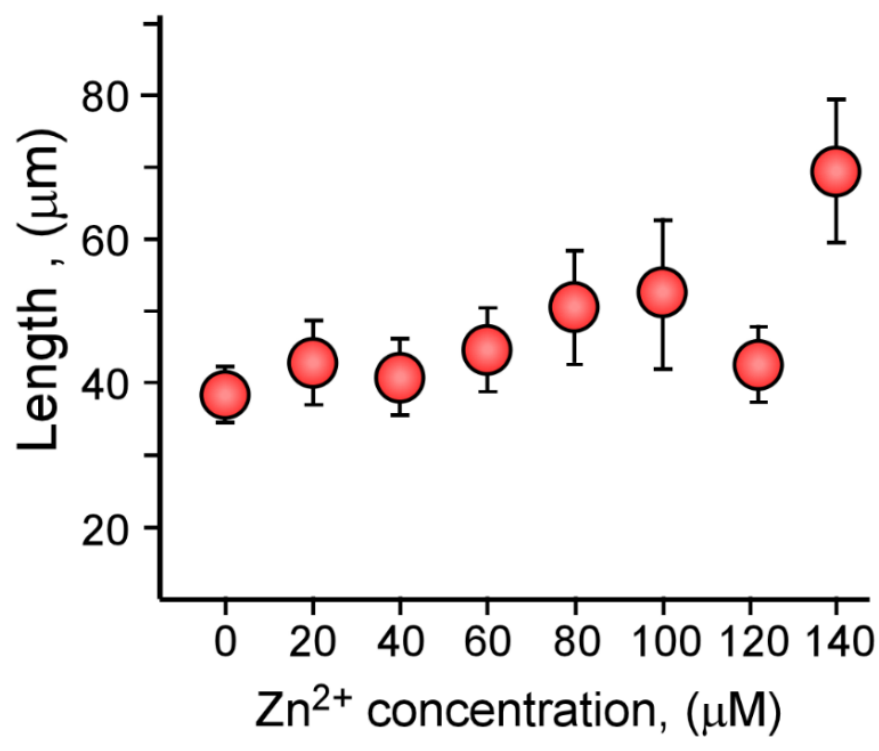

Figure S2. Average length of COM crystals (measured along the [001] direction) in the presence of $\mathrm{Zn}^{2+}$ ions. Symbols are the average of more than 500 measurements from a single batch. Error bars span two standard deviations. 


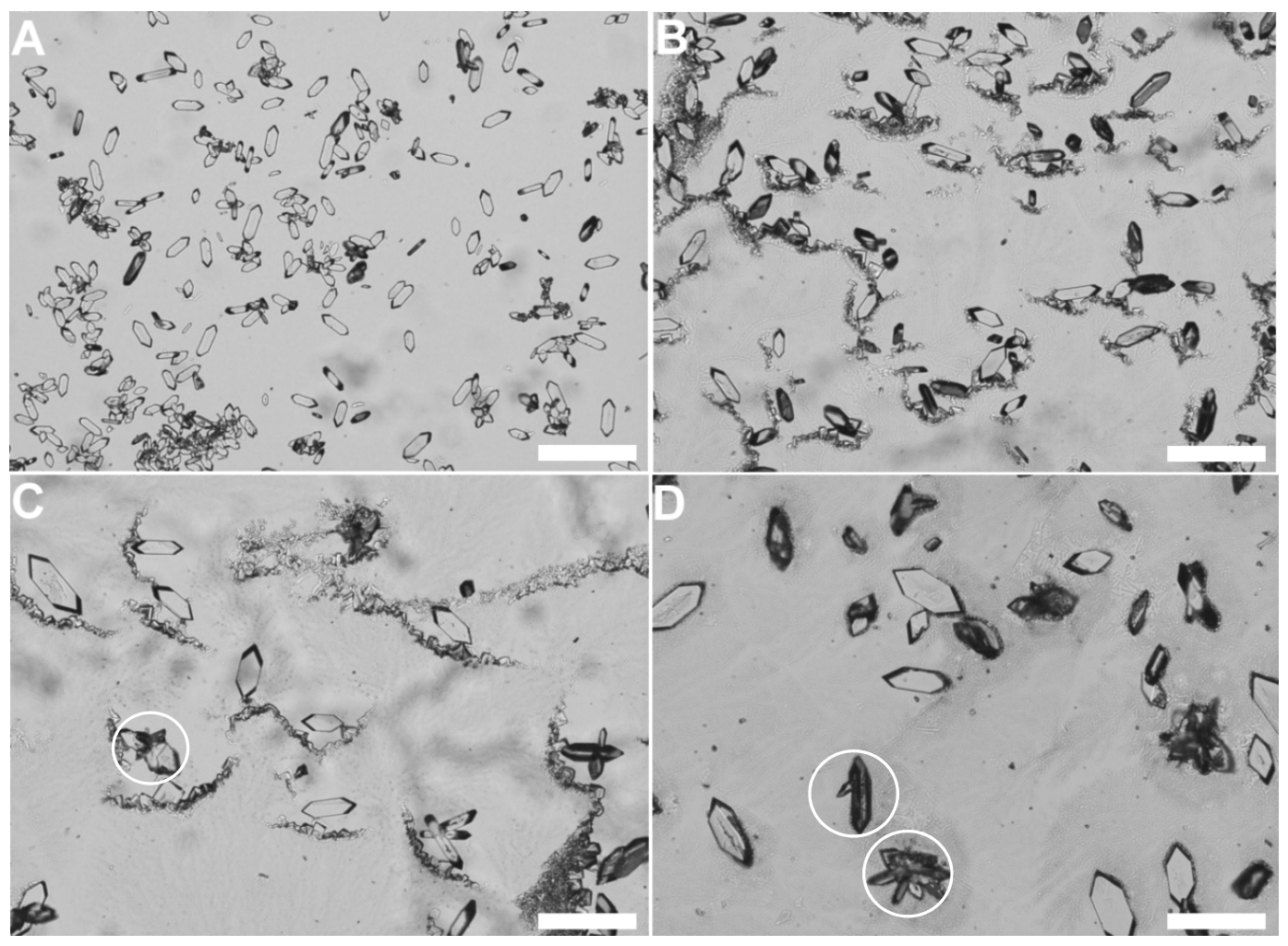

Figure S3. Optical micrographs showing the reduction in COM crystal number with increasing concentration of $\mathrm{Zn}^{2+}$ as (A) 0, (B) 40, (C) 100, and (D) $140 \mu \mathrm{M}$. Circles highlight the fraction of crystals with intergrowths. Scale bars are $100 \mu \mathrm{m}$.

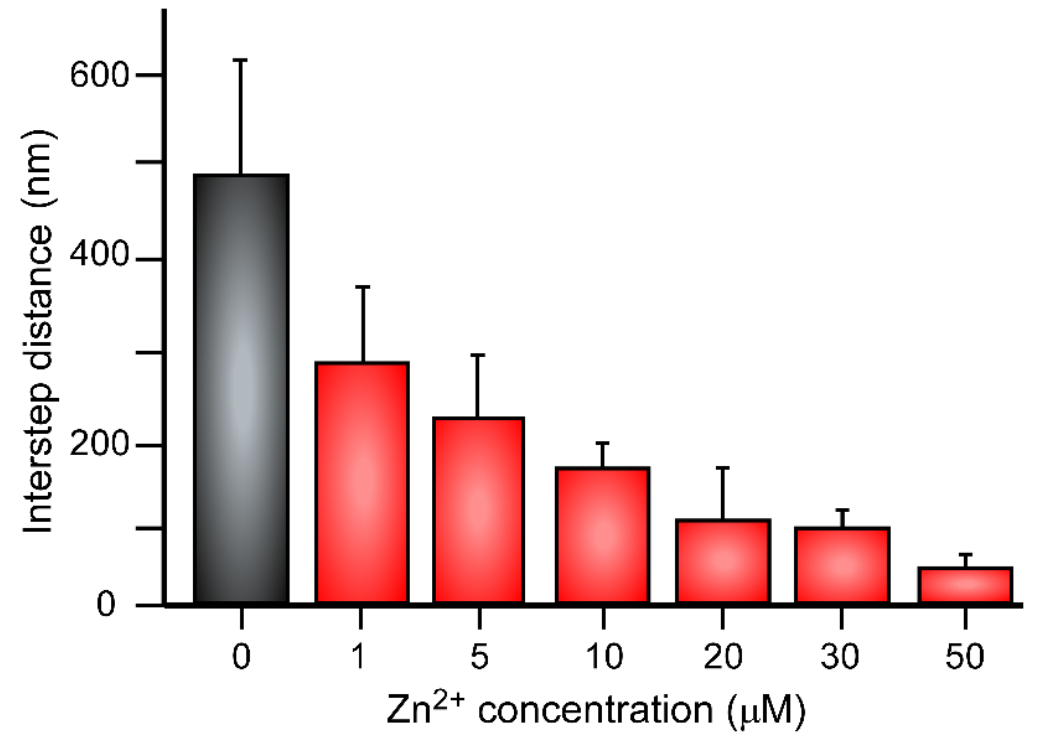

Figure S4. The average interstep distance measured by AFM on the basal surface of a COM (100) crystal decreases as the concentration of $\mathrm{Zn}^{2+}$ increases. Averages are based on more than 40 measurements from a single batch. Error bars equal one standard deviation. 


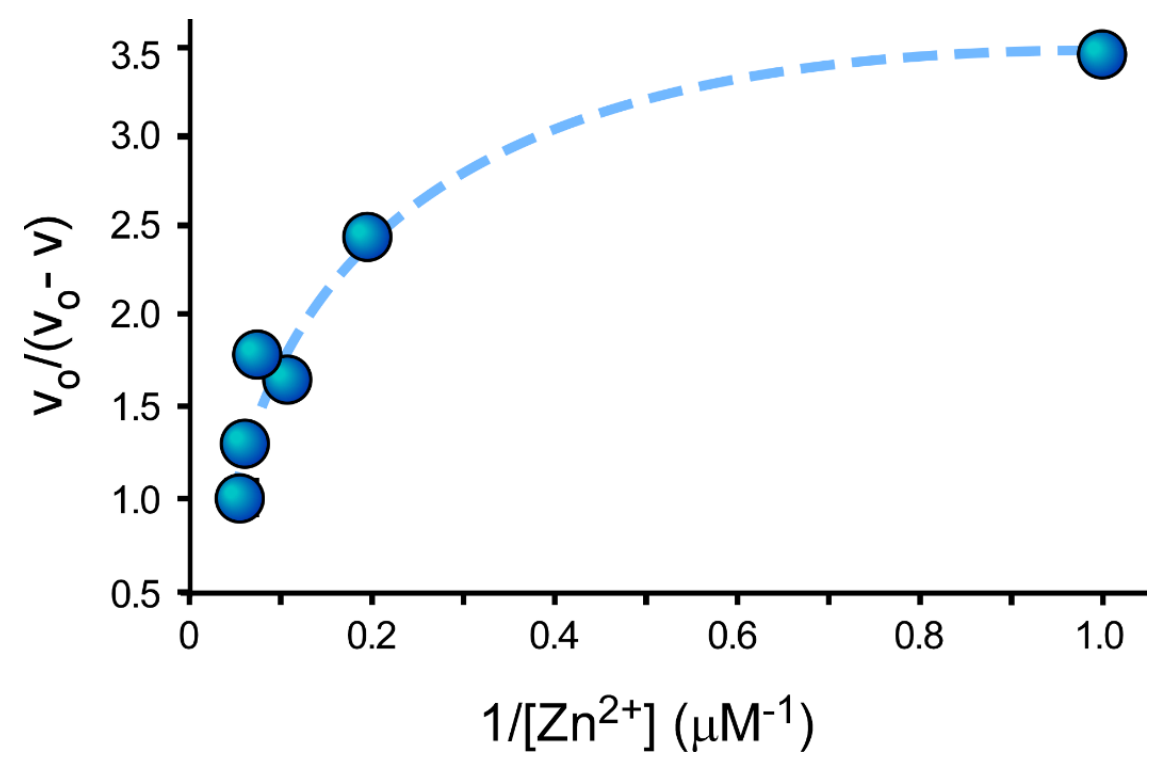

Figure S5. Bliznakov plot based on a reported $\operatorname{method}^{9}$ for COM step velocity as function of $\mathrm{Zn}^{2+}$ concentration. The nonlinearity of this plot indicates that $\mathrm{Zn}^{2+}$ does not act by a kinkblocking mechanism, but rather by step-pinning. The dashed line is interpolated to guide the eye.

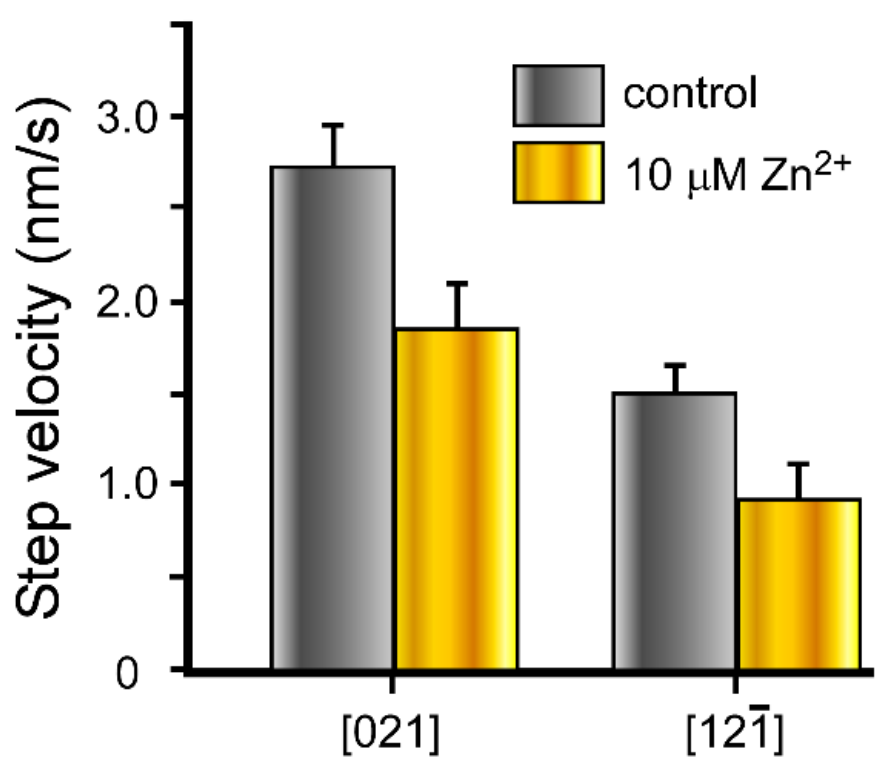

Figure S6. Step velocity measured by in situ AFM on a COM (010) surface corresponding to layer advancement along the [021] direction (left) and [12-1] direction (right). Values are compared for measurements in the absence (gray) and presence (yellow) of $10 \mu \mathrm{M} \mathrm{Zn}^{2+}$. Each data point is the average of more than 5 measurements from a single AFM experiment. Error bars equal one standard deviation. 


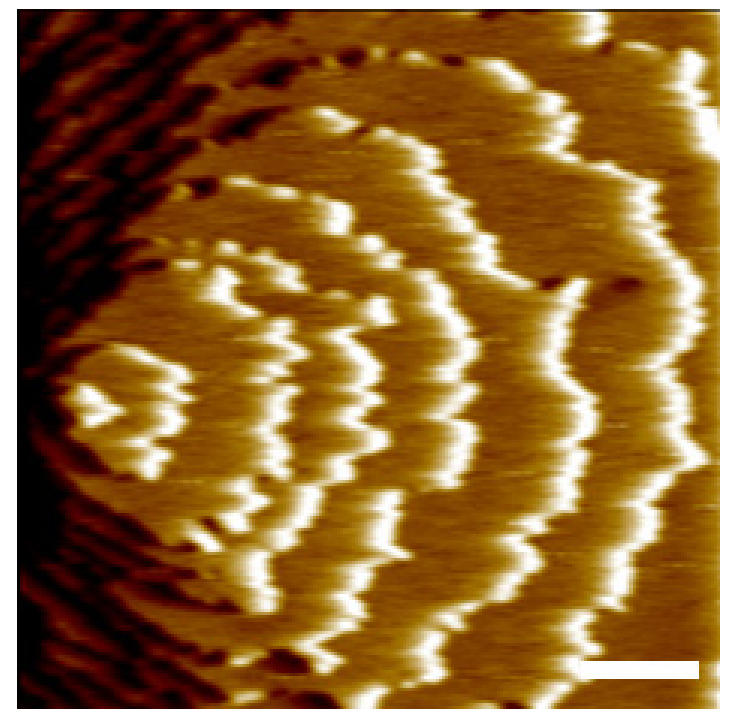

Figure S7. A representative AFM deflection mode image showing a COM (100) growth

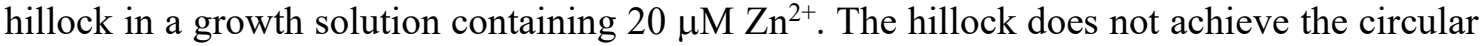
morphology observed for measurements in growth solutions with higher zinc content (e.g. 50

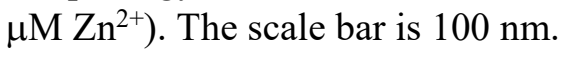

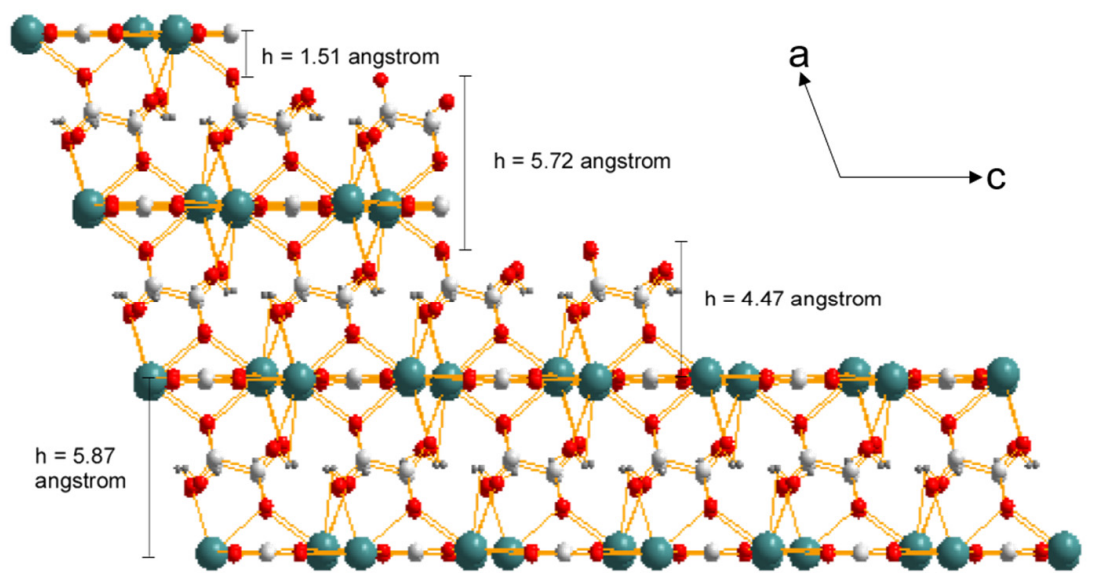

Figure S8. Visualization of COM terminations viewed along the (010) plane. The atoms are color coded as green (calcium), light gray (carbon), red (oxygen), and dark gray (hydrogen). This image was generated with the software Diamond. 

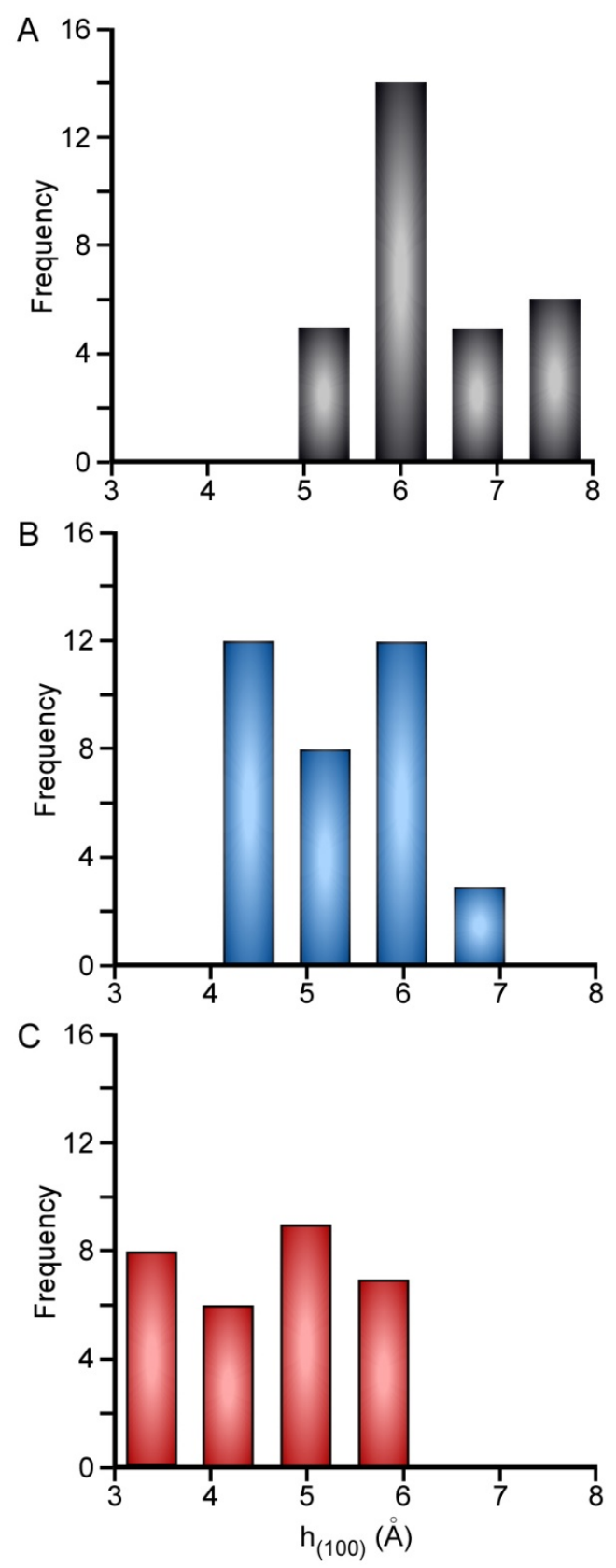

Figure S9. Step heights on COM (100) surfaces obtained from AFM measurements during growth experiments in the presence of (A) $0 \mu \mathrm{M} \mathrm{Zn}{ }^{2+}$ (control), (B) $20 \mu \mathrm{M} \mathrm{Zn}^{2+}$, and (C) 50 $\mu \mathrm{M} \mathrm{Zn}{ }^{2+}$. 

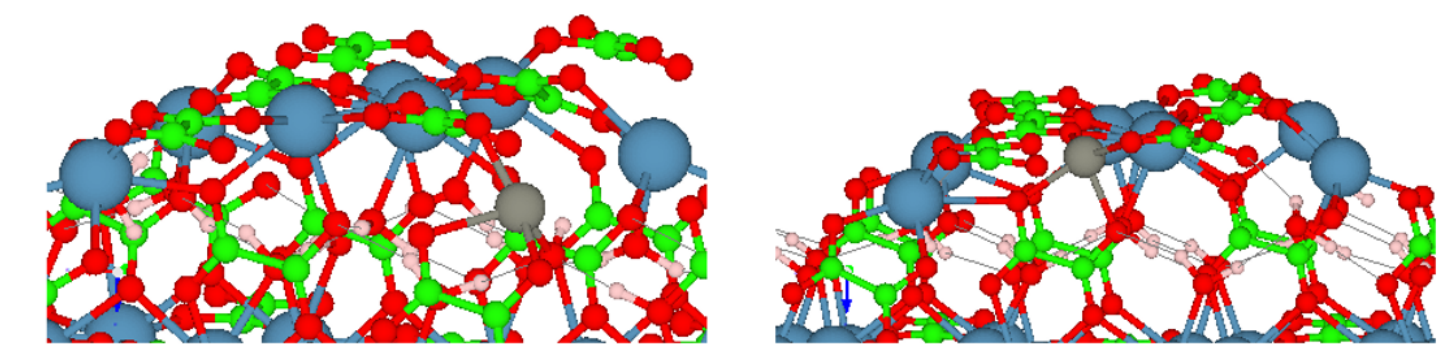

2

6

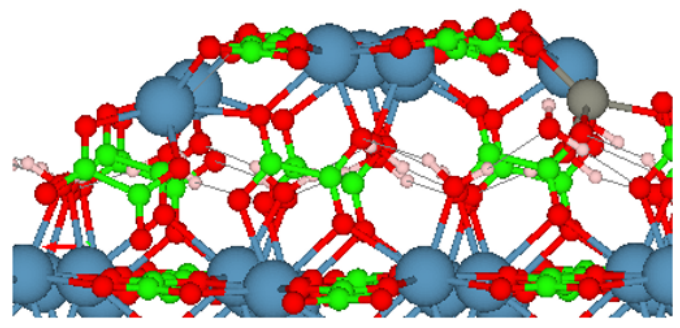

3

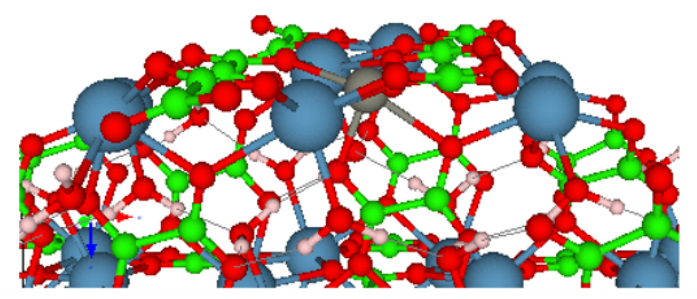

7
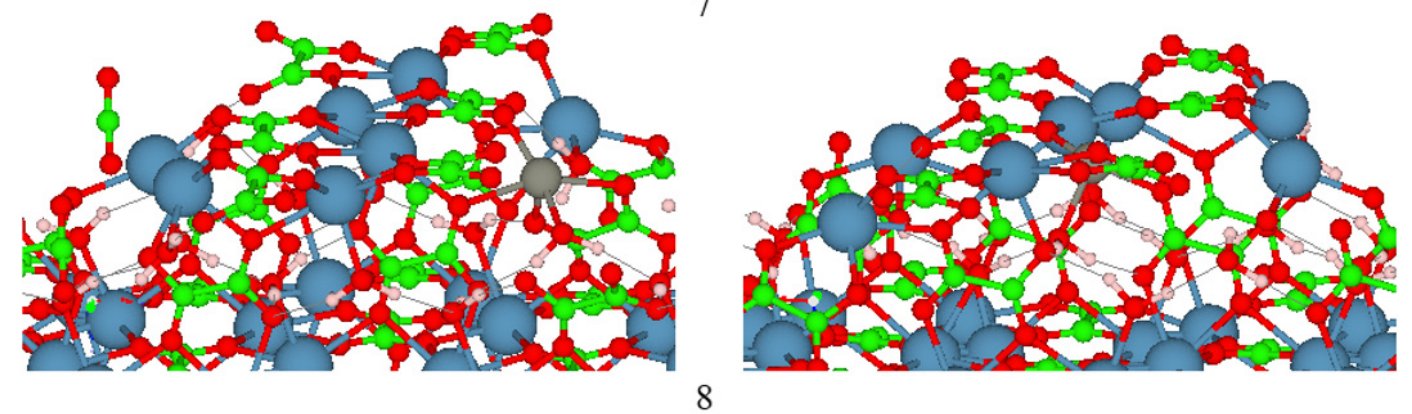

8
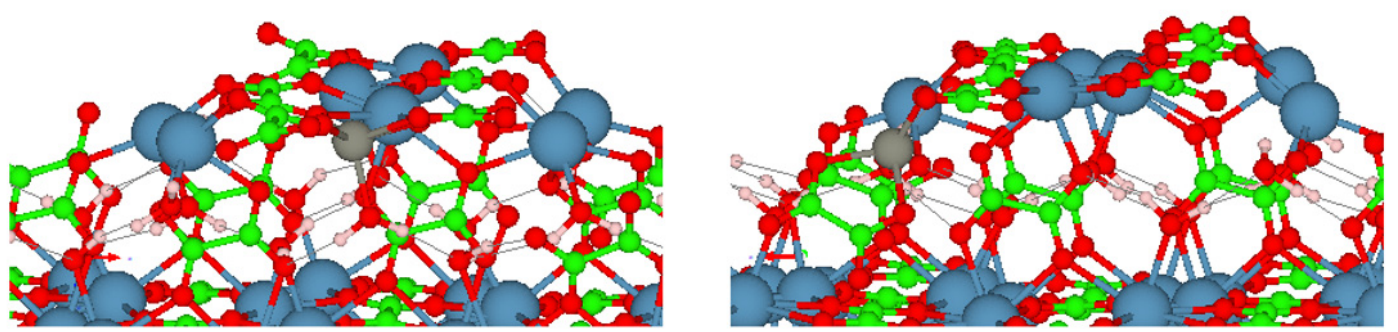

Figure S10: Optimized structures for a $\mathrm{Zn}$ defect in different step sites on the COM (100) surface as computed at the BLYP-D3/TZ2P level of theory. Here, elements Zn, Ca, C, O and $\mathrm{H}$ are colored gray, blue, green, red, and pink, respectively. Dashed lines indicate hydrogen bonds. The periodic direction for the step runs approximately from front to back. Site numbers correspond to those given in Table S1. All images were generated with the VESTA package. ${ }^{10}$ 


\section{SUPPORTING TABLES}

Table S1: Defect energetics, as computed at the BLYP-D3/TZ2P level of theory, for zinc substitution for calcium at each of the surface sites on the COM (100) surface with steps in the [001] direction. All values are given relative to the ions in aqueous solution based on the use of experimental hydration free energies.

\begin{tabular}{lc}
\hline Defect site & $\begin{array}{c}\text { Defect Energy } \\
\text { (relative to aqueous solution) } \\
\text { (kJ/mol) }\end{array}$ \\
\hline Bulk & +18.5 \\
Step edge site 1 & -153.5 \\
Step edge site 2 & -58.2 \\
Step edge site 3 & +69.7 \\
Step edge site 4 & -66.9 \\
Step edge site 5 & -73.4 \\
Step edge site 6 & -101.1 \\
Step edge site 7 & -78.8 \\
Step edge site 8 & -77.2 \\
\hline
\end{tabular}

\section{MOVIES}

Movie S1. Time-elapsed deflection mode images of a COM (100) surface during in situ AFM measurements using a supersaturated calcium oxalate growth solution without the addition of zinc. The total duration of the movie is $1.50 \mathrm{~min}$, captured at $78 \mathrm{~Hz}$. Scale bar is $100 \mathrm{~nm}$.

Movie S2. Time-elapsed deflection mode images of a COM (100) surface during in situ AFM measurements using a supersaturated calcium oxalate growth solution with the addition of 50 $\mu \mathrm{M} \mathrm{Zn}{ }^{2+}$. The total duration of the movie is $2.3 \mathrm{~min}$, captured at $78 \mathrm{~Hz}$. Scale bar is $100 \mathrm{~nm}$.

Movie S3. In situ AFM measurement of growth hillock recovery on a COM (100) surface after

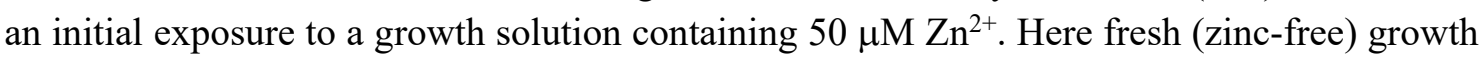
solution is introduced into the liquid cell. Continuous imaging reveals a transition from initial round layers (induced by the presence of zinc ions) into the anisotropic (tear drop shaped) layers that are characteristic of those observed for the control in Movie S1. The total duration of the movie is $1.5 \mathrm{~min}$, captured at $78 \mathrm{~Hz}$. Scale bar is $100 \mathrm{~nm}$. 


\section{REFERENCES}

1. Hutter, J.; Iannuzzi, M.; Schiffmann, F.; VandeVondele, J., cp2k: atomistic simulations of condensed matter systems. WIREs Computational Molecular Science 2014, 4 (1), 1525 .

2. VandeVondele, J.; Krack, M.; Mohamed, F.; Parrinello, M.; Chassaing, T.; Hutter, J., Quickstep: Fast and accurate density functional calculations using a mixed Gaussian and plane waves approach. Comput. Phys. Commun. 2005, 167 (2), 103-128.

3. Goedecker, S.; Teter, M.; Hutter, J., Separable dual-space Gaussian pseudopotentials. Phys. Rev. B 1996, 54 (3), 1703-1710.

4. Lee, C.; Yang, W.; Parr, R. G., Development of the Colle-Salvetti correlation-energy formula into a functional of the electron density. Phys. Rev. B 1988, 37 (2), 785-789.

5. Grimme, S.; Antony, J.; Ehrlich, S.; Krieg, H., A consistent and accurate ab initio parametrization of density functional dispersion correction (DFT-D) for the 94 elements H-Pu. J. Chem. Phys. 2010, 132 (15), 154104.

6. Tazzoli, V.; Domeneghetti, C., The crystal structures of whewellite and weddellite: reexamination and comparison. Am. Mineral. 1980, 65 (3-4), 327-334.

7. Fleming, S.; Rohl, A., GDIS: a visualization program for molecular and periodic systems. Z. Krist-Cryst. Mater. 2005, 220 (5-6), 580-584.

8. Marcus, Y., Thermodynamics of solvation of ions. Part 5.- Gibbs free energy of hydration at 298.15 K. J. Chem. Soc., Faraday Trans. 1991, 87 (18), 2995-2999.

9. Olafson, K. N.; Ketchum, M. A.; Rimer, J. D.; Vekilov, P. G., Mechanisms of hematin crystallization and inhibition by the antimalarial drug chloroquine. Proc. Natl. Acad. Sci. USA 2015, 112 (16), 4946.

10. Momma, K.; Izumi, F., VESTA 3 for three-dimensional visualization of crystal, volumetric and morphology data. J. Appl. Crystallogr. 2011, 44 (6), 1272-1276. 